\title{
Epicardial ablation of left ventricular arrhythmias originating from the left ventricular summit area without fluoroscopy
}

\author{
Guram Imnadze ${ }^{1} \odot$, Thomas Zerm ${ }^{2 *}$, Mustapha El Hamriti ${ }^{1}$, Leonard Bergau ${ }^{1}$, \\ Martin Braun ${ }^{1}$, Moneeb Khalaph ${ }^{1}$, Christian Sohns ${ }^{1}$, Philipp Sommer ${ }^{1}$ \\ ${ }^{1}$ Clinic for Electrophysiology, Herz- und Diabeteszentrum NRW, \\ Ruhr-University Bochum, Bad Oeynhausen, Germany \\ ${ }^{2}$ Department of Electrophysiology, Albertinen Hospital, Hamburg, Germany
}

\section{Introduction}

Ventricular arrhythmias originating from the left ventricular (LV) summit can be challenging [1]. The LV summit region can be reached from inside the great cardiac vein (GCV) and anterior interventricular vein [2]. Consequently, general interest in ablation techniques utilizing three-dimensional (3D) navigation systems without fluoroscopy increases according the ALARA "as low as reasonably achievable" principle [3]. Previous data demonstrated that ablation of supraventricular and ventricular tachycardias can be safely and effectively performed without the guidance of fluoroscopy [4-7]. This manuscript addresses technical issues in terms of epicardial catheter navigation within the great cardia vein and successful ablation of ventricular arrhythmias originating from the LV summit without the use of radiation.

\section{Current situation according to published literature}

Once faced with LV summit arrhythmia, we have to map carefully all possible access sites to this region: right ventricular outflow tract (RVOT), left ventricular outflow tract (LVOT), coronary cusp, and distal coronary sinus (CS) venous system/great cardiac vein. LV-summit arrhythmia fre- quently should be targeted from two or three of the above-mentioned sites. There is a lack of information about zero-fluoroscopy ablation of this region using a 3D navigation system only. Nevertheless, the available publications about limited- to zero-fluoroscopy of idiopathic ventricular arrhythmias can be helpful in understanding the topic.

Wang et al. [8] presented the largest cohort of patients with idiopathic ventricular arrhythmia in a multicenter study ablated with conventional ( $\mathrm{n}=326)$ and zero-fluoroscopy $(\mathrm{n}=163)$ technique. $94.4 \%$ of patients were successfully treated with a non-fluoroscopy approach. The only reason for switching to fluoroscopy (5.6\%) was the need for coronary angiography. There were no significant differences in terms of success rate, arrhythmia recurrence, and complications, but detailed information of the arrhythmia origin was not provided by this study [8].

Coronary angiography for visualization of the anatomical proximity of the ablation target to the coronary artery is a standard procedure during epicardial ablation of the LV-summit through the GCV. Steven et al. [9] showed that ablation of idiopathic ventricular arrhythmias can safely be performed from the GCV without significant permanent coronary artery stenosis. Moreover, the coronary spasms showed complete regression over time [9].

Address for correspondence: Univ. Prof. Dr. med. Philipp Sommer, Director Clinic for Electrophysiology, Herz- und DIabeteszentrum NRW, Ruhr-University Bochum, Georgstr. 11, D-32545 Bad Oeynhausen, Germany, tel: +49573197 1327, e-mail: gimnadze@hdz-nrw.de

Received: 2.02.2021 Accepted: 7.10.2021 Early publication date: 18.10.2021

*Equal contribution with the first author.

This article is available in open access under Creative Common Attribution-Non-Commercial-No Derivatives 4.0 International (CC BY-NC-ND 4.0) license, allowing to download articles and share them with others as long as they credit the authors and the publisher, but without permission to change them in any way or use them commercially. 
Today many centers are using different advanced imaging tools (integration of $3 \mathrm{D}$ reconstructed computed tomography [CT] or magnetic resonance imaging data or intracardiac echocardiography) for this purpose. Notably, in $5-6 \%$ of zero fluoroscopy cases rescue fluoroscopy is still required [10]. Some colleagues completely abandon coronary angiography, but there are no published data concerning the safety aspects of this approach.

Recently Rivera et al. [7] published the largest series of zero-fluoroscopic catheter ablation of arrhythmias with origin at the summit of the left ventricle. They used intracardiac echocardiography (ICE) for the verification of the safe distance between the ablation point and surrounded coronary arteries [7]. Indeed, it is an elegant solution for non-fluoroscopic technique, but there are still problems with reimbursement in many countries when using ICE.

Karkowski et al. [11] compared zero-fluoroscopy $(\mathrm{n}=88)$ with conventional $(\mathrm{n}=40)$ ablation strategies for the treatment of right- and left-sided ventricular arrhythmias. One third of the patients in the zero-fluoroscopy group underwent ablation of left-sided ventricular arrhythmias, which were equally distributed into three regions: the aortic cusp, LVOT, and left ventricle. The authors demonstrated that the zero-fluoroscopy approach is safe and equally effective compared to the conventional technique, and it should be implemented as often as possible for protection of patients and electrophysiologycal lab staff [11].

We report two successful epicardial ablation approaches for LV summit ventricular tachycardias (VT) with two different 3D mapping systems, without the use of fluoroscopy or other imaging tools.

\section{Case 1}

A 77-year-old male patient with frequent polymorphic premature ventricular contractions (PVCs) and episodes of non-sustained VT with history of aortic valve replacement and coronary artery bypass graft (CABG) surgery was admitted to our Electrophysiology Department. A 12-lead electrocardiogram (ECG) demonstrated a left antero-basal morphology of PVCs (Fig. 1). The ablation procedure was conducted using a contact 3D-mapping system (Carto System ${ }^{\circledR}$, Biosense Webster Inc.) and irrigated ablation catheter (4-mm tip) (SmartTouch $\mathrm{D} / \mathrm{F}^{\circledast}$, Biosense Webster Inc.). The earliest activation $(-20 \mathrm{~ms})$ was observed at the septal aspects of the RVOT. The attempt of radiofrequency (RF) ablation with $35 \mathrm{~W}$ at this point did not suppress the PVCs. Activation mapping distally inside the GCV showed the earliest activation (-33 ms). The pacemapping approach failed in the GCV (see Fig. 1). After two RF applications (with energy titration in the range 15-18-20 W, contact force $15-20 \mathrm{~g}$, maximal $30 \mathrm{~s}$ at one point) the PVCs were suppressed. No PVC recurrence was reported after a follow-up period of more than 1 year.

\section{Case 2}

A 74-year-old male patient was admitted to our hospital because of frequent monomorphic PVCs with bi- and trigemini pattern, as well as couplets, triplets, and non-sustained VTs. The LV ejection fraction (LVEF) was calculated to be $45-50 \%$. The diagnostic coronary angiography ruled out a relevant coronary heart disease, but pronounced allergic reaction (despite prophylaxis) to the contrast agent was noted. The procedure was conducted with a 3D-mapping system (NavX Precision, Abbott). An irrigated 4-mm tip ablation catheter (Flexibility D/F, Abbott) was utilized for advanced mapping and ablation (Fig. 2). The activation map during the PVC episodes of the RVOT and LVOT structures did not reveal the optimal site for ablation. Activation mapping during the PVC episodes showed the earliest activation $(-60 \mathrm{~ms})$ distal in the GCV with long fragmented pre-potentials. Pacemapping was not possible at this location. Three RF applications with energy titration (in the range 15-18-20 W) with a total duration of $80 \mathrm{~s}$ were applied at this zone. The PVCs were suppressed immediately after the second RF application. One-year follow-up showed freedom from any PVC recurrence, and the LVEF improved to $54 \%$.

\section{Discussion}

The epicardial origin of these arrhythmias is frequently located at the LV summit. The GCV divides the LV summit into two parts and might be accessible with an RF catheter [1]. Unfortunately, ablation in this region is not always possible due to several anatomical considerations [2]. Therefore, it might be beneficial to gain additional information about the individual anatomical relationship between the coronary arteries and cardiac veins from pre- and post-procedural coronary angiography [12].

We report two successful epicardial ablation approaches for LV summit VTs without the use of fluoroscopy with two different 3D mapping systems. This emphasizes that the current generation of $3 \mathrm{D}$ electroanatomical mapping systems allows 


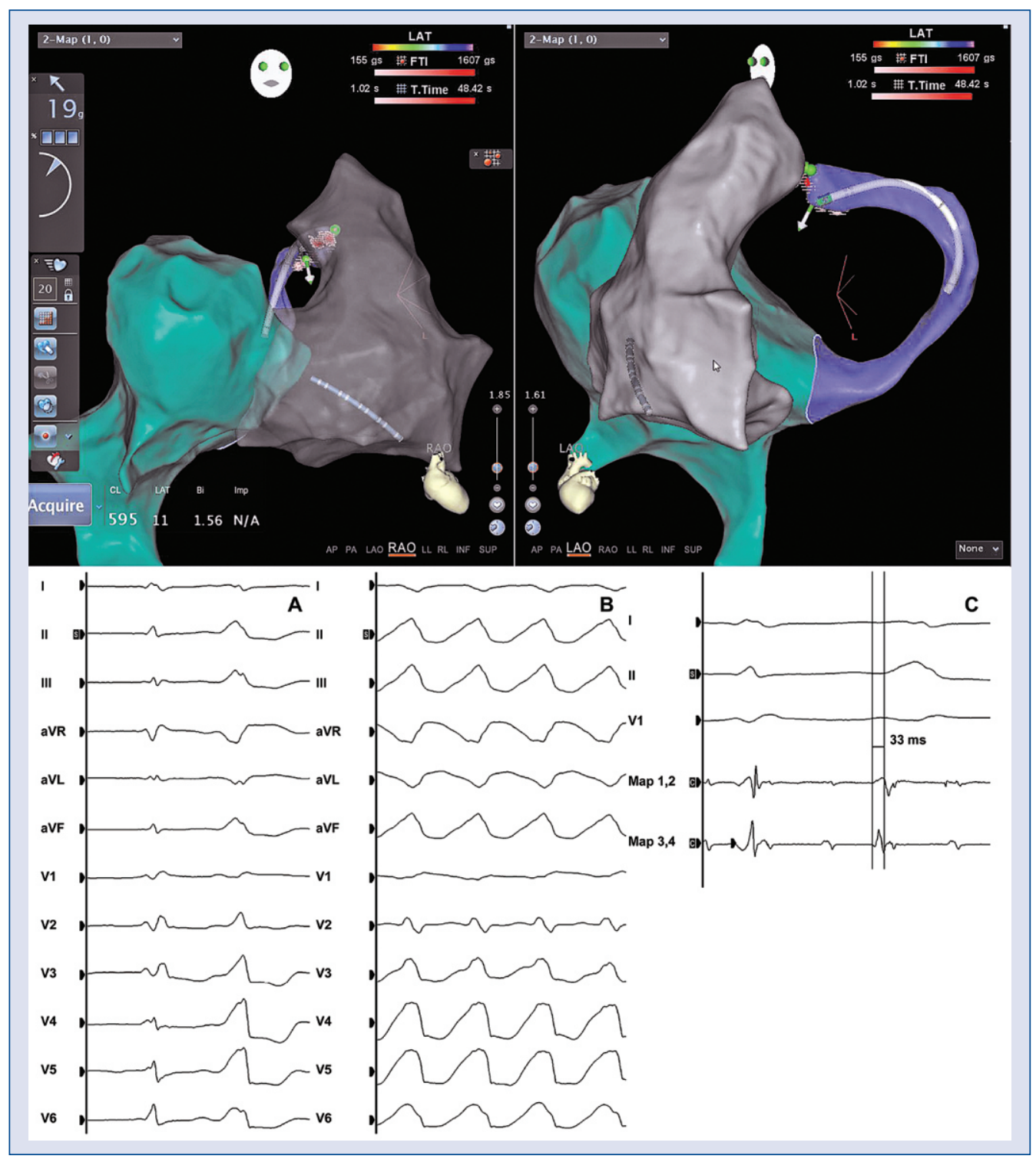

Figure 1. Top: Three-dimensional anatomical reconstruction (patient no. 1) of the inferior vena cava and right atrium (cyan), right ventricle (RV), and right ventricular outflow tract (RVOT) (gray), coronary sinus and great cardiac vein (violet). Left panel: Right anterior oblique (RAO) projection, RV, and RVOT with high transparency. Right panel: Left anterior oblique (LAO) projection. Bottom: 12-lead electrocardiogram of patient no. 1. A. QRS-morphology of the premature ventricular contractions; B. Sustained ventricular tachycardias during electrophysiologycal study; C. Earliest activation in the distal great cardiac vein.

for precise and safe navigation within complex cardiac structures without the general need for radiation.

The zero-fluoroscopy approach protects the patients and the staff, and offers new perspectives. For example, Mueller-Leisse et al. [13] suggested that many young women are concerned about radiation exposure, and this issue may explain the low number of female colleagues in interventional cardiology. The non-fluoroscopic approach is attractive also in this sense [13].
Due to the history of CABG we omitted another pure diagnostic coronary angiography in the first case. In the second case, coronary angiography was not performed because of two important observations: 1 ) the previous severe anaphylactic response to contrast agents (even despite prophylaxis) and 2) the epicardial activation map in the LV summit area found a very early, fragmented local activation, indicating extremely close proximity to the arrhythmia focus. Moreover, we used careful energy titration in both cases. Despite the report 


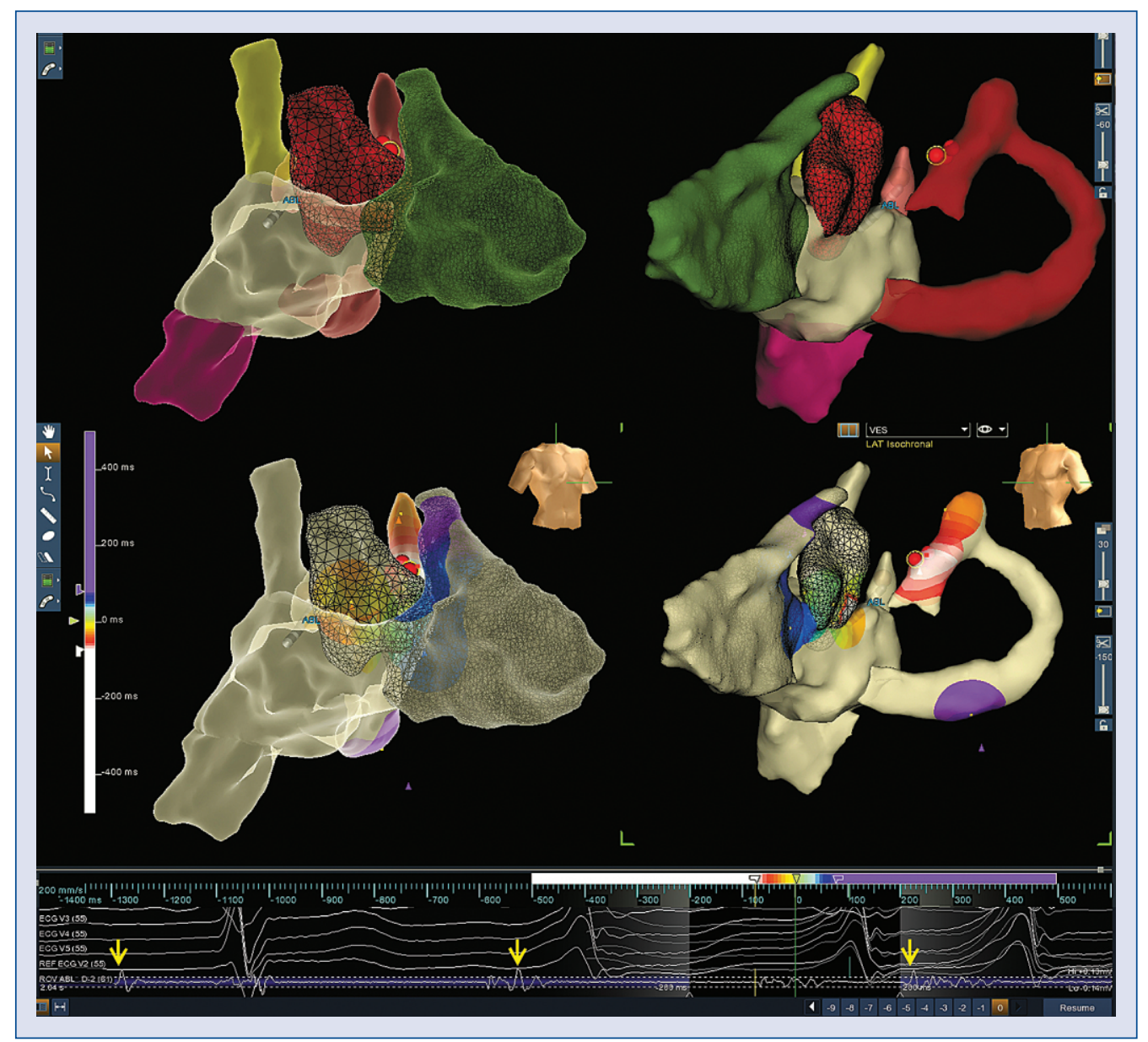

Figure 2. Top: Three-dimensional anatomical reconstruction (patient no. 2) of the cardiac structures in left-right anterior oblique (RAO) and right-left anterior oblique (LAO) projections. Inferior vena cava - purple, superior vena cava - yellow, right ventricle - green, persistent foramen ovale - catheter shadow with small piece of the left atrium — pink, coronary sinus and great cardiac vein — red, aortic cusp-mashed — dark red. Middle: The activation map, same projections of the same anatomical structures, white color indicates the earliest activation. Bottom: The electrocardiogram and intra-cardiac tracing from the ablation catheter at the ablation point (red points) shows earliest activation during the premature ventricular contractions. The yellow arrow indicates the atrial activation.

of straightforward procedures in this manuscript we do not want to suggest a general adoption of catheter navigation and ablation without the guidance of fluoroscopy.

\section{Conclusions}

Epicardial ablation of ventricular arrhythmias originating from the LV summit area without the need of fluoroscopy guided only with 3D navigation systems can be safely and effectively performed only under special anatomical and clinical conditions. Good anatomical knowledge and standardized mapping approaches together with stabile sedation conditions are major requirements for successful procedures without complications. Ideally, the procedure should be guided by a previous coronary angiography or by using modalities with pre- or periprocedural image integration in 3D mapping systems (X-ray, CT, or ICE). A careful titration of the RF energy seems to be beneficial.

Conflict of interest: Philipp Sommer reported as an Advisory Board Member for Abbott, Biosense Webster, Boston Scientific and Medtronic. Other authors declare no conflicts of interest.

\section{References}

1. Yamada T, McElderry HT, Doppalapudi H, et al. Idiopathic ventricular arrhythmias originating from the left ventricular summit: anatomic concepts relevant to ablation. Circ Arrhythm Electrophysiol. 2010; 3(6): 616-623, doi: 10.1161/CIRCEP.110.939744, indexed in Pubmed: 20855374. 
2. Nagashima K, Choi EK, Lin KY, et al. Ventricular arrhythmias near the distal great cardiac vein: challenging arrhythmia for ablation. Circ Arrhythm Electrophysiol. 2014; 7(5): 906-912, doi: 10.1161/CIRCEP.114.001615, indexed in Pubmed: 25110163.

3. Andreassi MG, Piccaluga E, Guagliumi G, et al. Occupational health risks in cardiac catheterization laboratory workers. Circ Cardiovasc Interv. 2016; 9(4): e003273, doi: 10.1161/CIRCINTERVENTIONS.115.003273, indexed in Pubmed: 27072525.

4. Lamberti F, Di Clemente F, Remoli R, et al. Catheter ablation of idiopathic ventricular tachycardia without the use of fluoroscopy. Int J Cardiol. 2015; 190(11): 338-343, doi: 10.1016/j. ijcard.2015.04.146, indexed in Pubmed: 25935624.

5. Imnadze G, Ajaj T, Bante H, et al. Transseptal puncture without fluoroscopy using a radiofrequency needle: A case series. Cardiol J. 2021; 28(5): 655-662, doi: 10.5603/CJ.a2020.0008, indexed in Pubmed: 32037502.

6. Akdeniz C, Elvin Gul E, Celik N, et al. Catheter ablation of idiopathic right ventricular arrhythmias in children with limited fluoroscopy. J Interv Card Electrophysiol. 2016; 46(3): 355-360, doi: 10.1007/s10840-016-0133-6, indexed in Pubmed: 27184808.

7. Rivera S, Vecchio N, Ricapito P, et al. Non-fluoroscopic catheter ablation of arrhythmias with origin at the summit of the left ventricle. J Interv Card Electrophysiol. 2019; 56(3): 279-290, doi: 10.1007/s10840-019-00522-1, indexed in Pubmed: 30729374.

8. Wang Y, Chen GZ, Yao Y, et al. Ablation of idiopathic ventricular arrhythmia using zero-fluoroscopy approach with equi- valent efficacy and less fatigue: a multicenter comparative study. Medicine (Baltimore). 2017; 96(6): e6080, doi: 10.1097/ MD.0000000000006080, indexed in Pubmed: 28178165.

9. Steven D, Pott C, Bittner A, et al. Idiopathic ventricular outflow tract arrhythmias from the great cardiac vein: challenges and risks of catheter ablation. Int J Cardiol. 2013; 169(5): 366-370, doi: 10.1016/j.ijcard.2013.09.008, indexed in Pubmed: 24182908.

10. Canpolat U, Faggioni M, Della Rocca DG, et al. State of fluoroless procedures in cardiac electrophysiology practice. J Innov Card Rhythm Manag. 2020; 11(3): 4018-4029, doi: 10.19102/ icrm.2020.110305, indexed in Pubmed: 32368376.

11. Karkowski G, Kuniewicz M, Koźluk E, et al. Non-fluoroscopic radiofrequency catheter ablation of right- and left-sided ventricular arrhythmias. Postepy Kardiol Interwencyjnej. 2020; 16(3): 321-329, doi: 10.5114/aic.2020.99268, indexed in Pubmed: 33597998.

12. Chen YH, Lin JF. Catheter ablation of idiopathic epicardial ventricular arrhythmias originating from the vicinity of the coronary sinus system. J Cardiovasc Electrophysiol. 2015; 26(10): 1160-1167, doi: 10.1111/jce.12756, indexed in Pubmed: 26175213.

13. Müller-Leisse J, Hillmann HA, Veltmann C, et al. [,Zero fluoro” - a chance for more women in electrophysiology]. Herzschrittmacherther Elektrophysiol. 2021; 32(2): 285-287, doi: 10.1007/ s00399-021-00767-2, indexed in Pubmed: 33944993. 